\title{
The Magnetic Leakage Inversion Method Based on Singular Value Decomposition of Magnetic Dipole Forward Model
}

\author{
Jieru Du, Yansong Li and Jun Liu \\ School of North China Electric Power University, Changping District, Beijing, 102206, China
}

\begin{abstract}
Ferromagnetic materials are widely used in many fields of national economy. In actual engineering, under the influence of stress or environment, ferromagnetic materials can be defective and have serious consequences. Therefore, magnetic flux leakage inversion, which is speculating defects information according to the detected magnetic leakage signals, is of great practical significance. In allusion to the identification of irregular defects, this paper presented an inversion method based on singular value decomposition of magnetic dipole forward model, which is very effective in identifying irregular defects. This paper contrasted and analyzed the distribution characteristics of magnetic intensity horizontal component $\boldsymbol{M x}$ when there was no defect and irregular defect, and the comparison verified that the magnetic intensity horizontal component $\boldsymbol{M x}$ could be used as an inversion gist. Then this paper presented the magnetic dipole forward model $\boldsymbol{B}=\boldsymbol{L} \boldsymbol{M}$. On account of the magnetic intensity component $\boldsymbol{M}$ containing defects information, this paper adopted the arithmetic of singular value decomposition of coefficient matrix $\boldsymbol{L}$ to solve the inversion equation $\boldsymbol{L} \boldsymbol{M}=\boldsymbol{B}$ and then acquired the distribution of magnetic intensity component $\boldsymbol{M}$. In the end, this paper verified the validity of this method.
\end{abstract}

\section{Introduction}

Ferromagnetic materials are widely used in various fields. However, the economic losses caused by the defects of ferromagnetic materials are numerous every year. Among them, magnetic flux leakage detection ${ }^{[1-2]}$ has been widely used in defect detection of ferromagnetic materials due to its advantages such as high sensitivity, low cost, fast speed, low requirements on workpiece surface cleanliness. Leakage magnetic inversion ${ }^{[3-4]}$, that is, the location and size of the defect are calculated according to the known leakage magnetic field signals, is the solution process of electromagnetic field inverse problem.

Currently, the most widely used inversion methods are neural network method and optimization algorithm. The neural network method includes BP neural network with arbitrary approximation accuracy ${ }^{[5]}$, RBF neural network with optimal approximation characteristics ${ }^{[6]}, \mathrm{WBF}$ neural network with hierarchical resolution and finite element neural network method. Based on iteration point data and objective function, optimization algorithm updates defect outline to make it close to the actual size. Optimization algorithm mainly includes the steepest descent method, conjugate gradient method, genetic algorithm, particle swarm optimization algorithm ${ }^{[7]}$, simulated annealing method and ant colony optimization algorithm, and different methods have different convergence rates.

Considering that the neural network method is highly dependent on samples and the finite element neural network method requires a large computational capacity, this paper presents a new magnetic flux leakage detection inversion method based on the singular value decomposition of magnetic dipole forward model. Singular value decomposition is a kind of perfect orthogonal decomposition with good properties. Its core is to give the effective rank of the matrix without changing the relative measurement characteristics of the matrix.

In this paper, the defect information is represented by magnetized intensity component, and the validity of this method is verified by different shape defects. This inversion method does not require prior information and does not need to consider the problem of convergence. What's more, it brings only a small amount of calculation and has high efficiency. Moreover, it is suitable for defects of various shapes and strong generalization ability is its prominent advantage.

\section{Comparison and Analysis of Magnetic Intensity}

To analyze the electromagnetic field distribution, this paper built the defect plate model firstly. With the help of COMSOL multiphysics simulation software, we can observe the distribution of magnetic intensity horizontal component $\boldsymbol{M x}$. During the course of magnetization, only the region between two magnetic poles can be

*Corresponding author:987509190@qq.com 
magnetized locally, therefore this area is called the effective detection area. When there is no defect and a triangle defect, the distribution is shown in Figure 1.

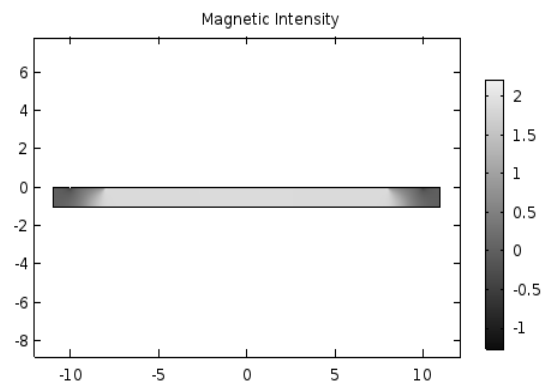

(a)distribution of $\boldsymbol{M x}$ when there is no defect

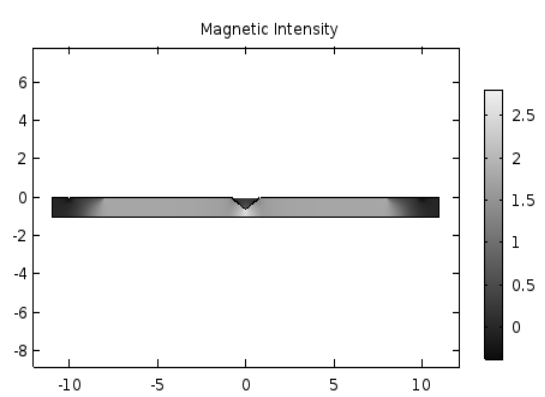

(b)distribution of $\boldsymbol{M x}$ when there is a triangle defect

Figure 1. Distribution of magnetic intensity horizontal component $\boldsymbol{M x}$.

As shown in Figure.1(a), when there is no defect, the magnetic intensity horizontal component $\boldsymbol{M x}$ in the effective detection area is distributed with large numerical value and it is symmetric about the center of the plate. While in Figure.1(b), the magnetic intensity horizontal component $\boldsymbol{M x}$ decreases suddenly in the defect area, and it is quite different from the other parts in the effective detection area of the plate. From the distribution differences, we can preliminary recognize the defect location, shape and size etc. This difference makes it possible that the magnetic intensity horizontal component $\boldsymbol{M} \boldsymbol{x}$ can be used as the gist of inversion. This is because air has a low permeability compared with ferromagnetic materials, thus it's difficult to magnetization. Under the same magnetization conditions, the magnetic intensity in the airshed is far less than that in the ferromagnetic materials.

\section{Magnetic Dipole Forward Model}

\subsection{Magnetic Dipaole Model}

The vector magnetic potential produced by a magnetic dipole is shown in Formula 1.

$$
\boldsymbol{A}=\frac{\mu_{0}}{4 \pi} \frac{\boldsymbol{m} \times \boldsymbol{e}_{\boldsymbol{R}}}{R^{2}}
$$

Among them, $\boldsymbol{m}$ is a magnetic dipole moment. Magnetic intensity is the vector sum of the magnetic dipole moments in a unit volume, and it is shown in Formula 2.

$$
\boldsymbol{M}=\lim _{\Delta V^{\prime} \rightarrow 0} \frac{\sum \boldsymbol{m}}{\Delta V^{\prime}}
$$

Then the vector sum of magnetic dipole moments in any volume element can be expressed as $\boldsymbol{M} d V^{\prime}$. The vector magnetic potential is as follows.

$$
\begin{gathered}
d \boldsymbol{A}=\frac{\mu_{0}}{4 \pi} \frac{\boldsymbol{M} \times \boldsymbol{e}_{\boldsymbol{R}}}{R^{2}} d V^{\prime} \\
\boldsymbol{B}=\nabla \times \boldsymbol{A}
\end{gathered}
$$

Based on the relationship, shown in Formula 4, between magnetic intensity $\boldsymbol{B}$ and vector magnetic potential $\boldsymbol{A}$, for the two-dimensional defect plate, the magnetic induction intensity $\boldsymbol{B}$ generated by all magnetic dipoles can be obtained.

$$
\begin{aligned}
\boldsymbol{B}= & \iint_{N^{\prime}} \frac{\mu_{0}}{4 \pi R^{5}}\left\{\left[3\left(x-x^{\prime}\right)^{2}-R^{2}\right] m_{x}+\right. \\
& \left.3\left(x-x^{\prime}\right)\left(y-y^{\prime}\right) m_{y}\right\} \boldsymbol{e}_{x} d S^{\prime}+ \\
& \iint_{N^{\prime}} \frac{\mu_{0}}{4 \pi R^{5}}\left\{3\left(x-x^{\prime}\right)\left(y-y^{\prime}\right) m_{x}+\right. \\
& {\left.\left[3\left(y-y^{\prime}\right)^{2}-R^{2}\right] m_{y}\right\} \boldsymbol{e}_{y} d S^{\prime} }
\end{aligned}
$$

\subsection{Numerical Integration Calculation Method}

When the integrand function on the the integrating range is complex, it is difficult to integrate analytically, so we usually adopt numerical integration method. The numerical integration method requires the integral unit should have a regular shape, therefore, it's suitable for a standard unit. The two-dimensional defect plate is divided into several quadrilateral units and the local coordinates are connected with the global coordinates by isoparametric transformation. In the local coordinate system, gauss integral formula on the standard integral unit is:

$$
\begin{aligned}
\int_{-1}^{1} \int_{-1}^{1} f(\alpha, \beta) d \alpha d \beta & =\int_{-1}^{1}\left[\int_{-1}^{1} f(\alpha, \beta) d \alpha\right] d \beta \\
& =\sum_{i=1}^{n 2} H_{i}\left[\sum_{j=1}^{n 1} H_{j} f\left(\alpha_{i}, \beta_{j}\right)\right] \\
& =\sum_{i=1}^{n 2} \sum_{j=1}^{n 1} H_{i} H_{j} f\left(\alpha_{i}, \beta_{j}\right)
\end{aligned}
$$

The leakage magnetic field in any point generated by any quadrilateral integral unit is:

$$
\begin{aligned}
& B_{n e, x}=\frac{\mu_{0}}{4 \pi} \sum_{i=1}^{n 2} \sum_{j=1}^{n 1} \frac{H_{i} H_{j}}{R_{i j}^{5}}\left\{\left[3\left(x-x_{i j}^{\prime}\right)^{2}-R_{i j}^{2}\right] D_{n_{e}, i j} M_{x i j}+\right. \\
&\left.3\left(x-x_{i j}^{\prime}\right)\left(y-y_{i j}^{\prime}\right) D_{n_{e}, i j} M_{y i j}\right\}=\frac{\mu_{0}}{4 \pi}\left[A\left(1, n_{e}\right) M_{x, n e}+C\left(1, n_{e}\right) M_{y, n e}\right] \\
& B_{n e, y}= \frac{\mu_{0}}{4 \pi} \sum_{i=1}^{n 2} \sum_{j=1}^{n 1} \frac{H_{i} H_{j}}{R_{i j}^{5}}\left\{3\left(x-x_{i j}^{\prime}\right)\left(y-y_{i j}^{\prime}\right) D_{n_{e}, i j} M_{x i j}+\right. \\
& {\left.\left[3\left(y-y_{i j}^{\prime}\right)^{2}-R_{i j}^{2}\right] D_{n_{e}, i j} M_{y i j}\right\} } \\
&=\frac{\mu_{0}}{4 \pi}\left[C\left(1, n_{e}\right) M_{x, n e}+B\left(1, n_{e}\right) M_{y, n e}\right] \\
& R_{i j}=\sqrt{\left(x-x_{i j}^{\prime}\right)^{2}+\left(\mathrm{y}-y_{i j}^{\prime}\right)^{2}}
\end{aligned}
$$

In the equation, " $x$ " and " $y$ " are the overall 
coordinates of the field point to be solved, and " $\mathrm{x}$ ' and " $y$ ' ${ }_{i j}$ " are the overall coordinates of the ne-th gauss integral point. $D_{n e, i j}$ is the transformation coefficient, by which the integral unit in the local coordinate is transformed into that in the overall coordinate. The two-dimensional defect plate is divided into $\mathrm{Ne}$ units, and the leakage magnetic field $\boldsymbol{B}$ at any point generated by the magnetized source area is deduced from Formula 7.

$$
\begin{aligned}
& B_{x}=\frac{\mu_{0}}{4 \pi} \sum_{n_{e}=1}^{N e}\left[A\left(1, n_{e}\right) M_{x, n e}+C\left(1, n_{e}\right) M_{y, n e}\right] \\
& B_{y}=\frac{\mu_{0}}{4 \pi} \sum_{n_{e}=1}^{N e}\left[C\left(1, n_{e}\right) M_{x, n e}+B\left(1, n_{e}\right) M_{y, n e}\right]
\end{aligned}
$$

Finally, we obtain the relationship (shown in Formula 10) between the magnetic induction intensity $\boldsymbol{B}$ and magnetic intensity $\boldsymbol{M}$.

$$
\left[\begin{array}{l}
B_{x} \\
B_{y}
\end{array}\right]=\left[\begin{array}{ll}
A & C \\
C & B
\end{array}\right]\left[\begin{array}{l}
M_{x} \\
M_{y}
\end{array}\right]
$$

With the help of COMSOL multiphysics simulation software, we obtain the value of leakage magnetic field and the comparison between simulation results and calculation results is shown in Figure 2 .

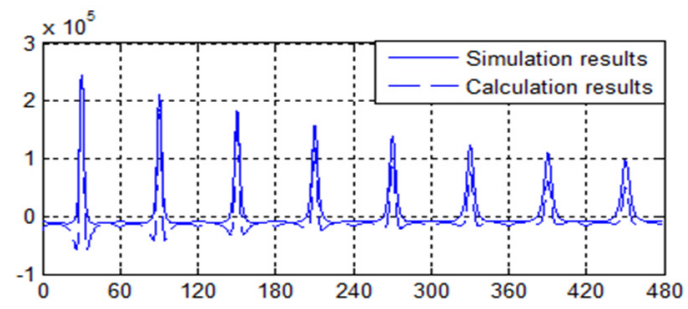

(a) comparison of $\boldsymbol{H} \boldsymbol{x}$ component

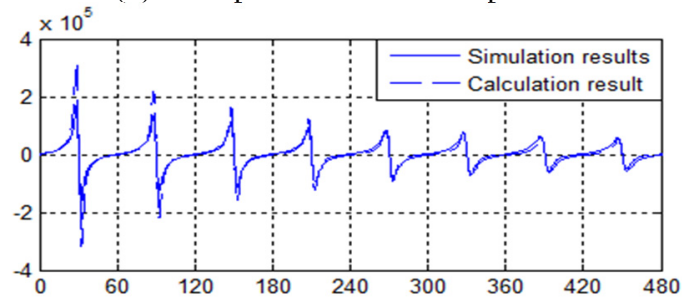

(b) comparison of $\boldsymbol{H} \boldsymbol{y}$ component

Figure 2.Comparison between simulation results and calculation result.

Considering the value of $\boldsymbol{M y}$ component is far less than $\boldsymbol{M} \boldsymbol{x}$ component, and it is basically distributed in a small area around the defect, therefore, we can ignore the effect of $\boldsymbol{M y}$ component, then we obtain a succinct formula.

$$
\left[\begin{array}{l}
B_{x} \\
B_{y}
\end{array}\right]=\left[\begin{array}{l}
A \\
C
\end{array}\right] M_{x}
$$

The effect of $\boldsymbol{M} \boldsymbol{y}$ component on $\boldsymbol{H} \boldsymbol{x}$ component and $\boldsymbol{H y}$ component is shown in Figure 3.

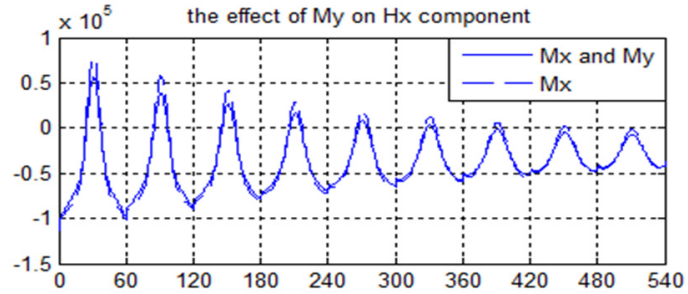

(a) the effect of $\boldsymbol{M} \boldsymbol{y}$ component on $\boldsymbol{H} \boldsymbol{x}$ component

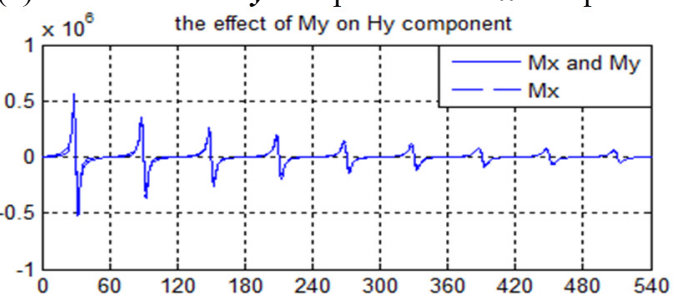

(b) the effect of $\boldsymbol{M y}$ component on $\boldsymbol{H} \boldsymbol{y}$ component Figure 3. The effect of $\boldsymbol{M} \boldsymbol{y}$ component.

\section{Inversion Algorithm}

According to the magnetic dipole forward model, the inversion question is shown in Formula 12.

$$
\boldsymbol{L} \boldsymbol{M}=\boldsymbol{B}
$$

In Formula $12, \boldsymbol{L}$ is a NP $\mathrm{x} \mathrm{Nd}$ dimension coefficient matrix. The number of measured field points is NP and that of nodes after grid division in the source area is $\mathrm{Nd}$. In general, the number of measurement points NP is much larger than that of the unknown variable $\mathrm{Nd}$, thus the solution is not unique. Therefore, this paper obtains the minimal norm solution which is single and it satisfies Formula 13.

$$
\|\boldsymbol{e}\|_{\min }=\left\|\boldsymbol{L} \boldsymbol{m}^{*}-\boldsymbol{b}\right\|
$$

The singular value decomposition of coefficient matrix $\boldsymbol{L}$ is used to obtain the minimum norm solution of the least square problem so as to satisfy the minimum error requirement. The singular value decomposition process of coefficient matrix $\boldsymbol{L}$ is shown as follows.

$$
\begin{gathered}
L=U X V^{T} \\
X V^{T} m=U^{T} b
\end{gathered}
$$

Suppose:

$$
\begin{gathered}
U^{T} b=q=\left[\begin{array}{l}
q_{1} \\
q_{2}
\end{array}\right] \\
V^{T}=s=\left[\begin{array}{l}
s_{1} \\
s_{2}
\end{array}\right]
\end{gathered}
$$

Among them, $\boldsymbol{q}_{1}$ and $\boldsymbol{s}_{\boldsymbol{1}}$ are $\mathrm{q} \times 1$ dimension vectors. Based on the above, we acquire Formula 18.

$$
X s=q
$$

Since $\boldsymbol{X}$ is a diagonal matrix, it can be directly solved as follows.

$$
\begin{aligned}
& {\left[\begin{array}{cc}
\boldsymbol{A}_{\boldsymbol{k} \times \boldsymbol{k}} & \mathbf{0} \\
\boldsymbol{0} & \boldsymbol{0}
\end{array}\right]\left[\begin{array}{l}
\boldsymbol{s}_{1} \\
\boldsymbol{s}_{2}
\end{array}\right]=\left[\begin{array}{l}
\boldsymbol{q}_{1} \\
\boldsymbol{q}_{2}
\end{array}\right]} \\
& A=\operatorname{diag}\left(\sigma_{1}, \sigma_{2}, \cdots \sigma_{k}\right)
\end{aligned}
$$




$$
\sigma_{1} \geq \sigma_{2} \geq \sigma_{3} \geq \cdots \sigma_{k} \geq 0
$$

$\sigma \mathrm{i}(1 \leq \mathrm{i} \leq \mathrm{k})$ is singular value of $\boldsymbol{L}$.

$$
A s_{1}^{*}=q_{1}
$$

Formula 22 has a unique solution. Since $\boldsymbol{s}_{2}$ can be taken arbitrarily, so $\boldsymbol{m}$ can be represented as Formula 23.

$$
\boldsymbol{m}=\boldsymbol{V}\left[\begin{array}{c}
s_{1}^{*} \\
s_{2}
\end{array}\right]
$$

Error calculation is shown in Formula 24.

$$
\boldsymbol{e}=\boldsymbol{L} \boldsymbol{m}-\boldsymbol{b}=\boldsymbol{U} X \boldsymbol{V}^{T} \boldsymbol{m}-\boldsymbol{b}=\boldsymbol{U}\left(\boldsymbol{X}\left[\begin{array}{l}
s_{1}{ }^{*} \\
s_{2}
\end{array}\right]-\boldsymbol{q}\right)
$$

As can be seen from Formula 24, when $\boldsymbol{S} \boldsymbol{2}=\boldsymbol{0}$, the result satisfying the minimum error is obtained.

$$
\boldsymbol{m}^{*}=\boldsymbol{V}\left[\begin{array}{c}
s_{1}^{*} \\
0
\end{array}\right]
$$

\section{Analysis of examples}

\subsection{Rectangular Defect}

Taken the two-dimensional magnetization model of defect leakage magnetic field as an example, the rectangular defect size is $0.4 \mathrm{~mm} * 0.6 \mathrm{~mm}$, located at the center of the plate. Considering the number of variables and the operation speed, the size of grid division in the source area is $0.05 \mathrm{~mm}$. The magnetic dipole model is established on the premise that the field point is far from the source area, but the magnetic leakage signals decrease greatly when the lifting height is too high, thus containing less defect information. Finally the lift height of cut lines is selected to $0.3 \mathrm{~mm}$ to $0.5 \mathrm{~mm}$. The length range of the cut lines is $-4.00 \mathrm{~mm}--3.98 \mathrm{~mm}$, in which the cut interval is $0.02 \mathrm{~mm}$, and 400 cut points are taken from each cut line. The two adjacent cut lines are separated by $0.05 \mathrm{~mm}$. 5 cut lines are taken for a total of 2000 field points.

The distribution of the magnetization intensity horizontal component $\boldsymbol{M x}$ in the source area is calculated by MATLAB software. The defect outline can be identified preliminarily. As shown in Figure 4, it can be preliminarily speculated that the defect shape is rectangular. The defect width range is $0.4 \mathrm{~mm}-0.5 \mathrm{~mm}$, and the depth range is $0.55 \mathrm{~mm}-0.60 \mathrm{~mm}$, which is relatively close to the authentic size of $0.4 \mathrm{~mm} * 0.6 \mathrm{~mm}$. The defect resolution is $0.05 \mathrm{~mm}$, which is consistent to the mesh size.

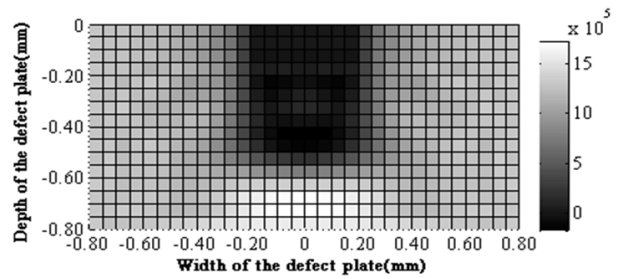

Figure 4. The inversion result of a rectangular defect.

\subsection{Triangular Defect}

In practical engineering, the most common crack defects have a narrow bottom and a wide top, which are similar to triangle defects. Located at the center of the defect plate, the bottom width of the triangle defect is $0.8 \mathrm{~mm}$, and the depth is $0.6 \mathrm{~mm}$. Through singular value decomposition inversion algorithm, the magnetization intensity distribution is shown in Figure 5. The triangular silhouette could be identified easily. The bottom width of triangle defect is $0.80 \mathrm{~mm}$, and the depth range is $0.55 \mathrm{~mm}-0.60 \mathrm{~mm}$, thus it can be seen that this algorithm is ideal for the inversion of triangular defects.

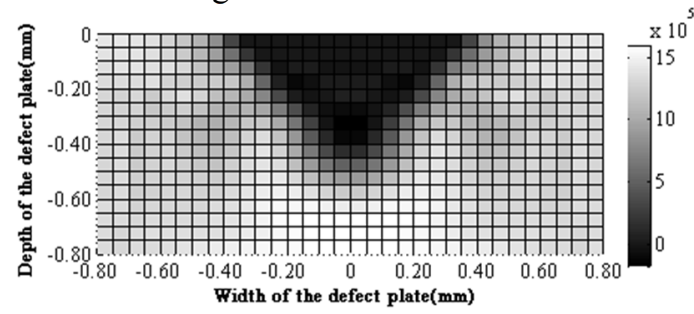

Figure 5. The inversion result of a triangular defect.

\subsection{Trapezoidal Defect}

Both of the above-mentioned defects have regular shapes. When the defect shape is an isosceles trapezoid with an upper bottom of $1.2 \mathrm{~mm}$, a lower bottom of $0.4 \mathrm{~mm}$ and a height of $0.4 \mathrm{~mm}$, the inversion result is shown in Figure 5. The defect profile can be clearly identified as isosceles trapezoid The upper bottom is $1.20 \mathrm{~mm}$, the lower bottom is $0.40 \mathrm{~mm}$, and the height is $0.40 \mathrm{~mm}-0.45 \mathrm{~mm}$, which is basically consistent with the actual size. The inversion result of trapezoid defect verifies that this singular value decomposition inversion algorithm is not applicable to regular defects, but has a good recognition effect for defects with complex shapes.

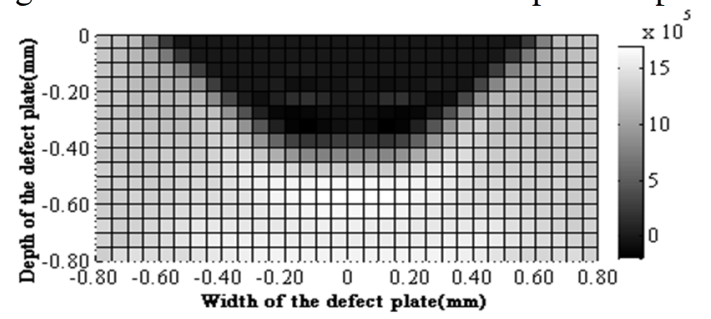

Figure 5. The inversion result of a trapezoidal defect.

\section{Conclusion}

This paper compared and analyzed the distribution characteristics of magnetic intensity horizontal component Mx firstly, and the comparison verified the validity of selecting $\mathrm{Mx}$ as inversion gist. Then this paper put forward the unit integral algorithm of magnetic dipole forward question, by which we could calculate the leakage magnetic field at any field point. Based on this forward model, we adopted the method of singular value decomposition of forward model coefficient matrix to acquire the magnetic intensity distribution which contains defect information. So far, the process of 
inversion was realized. Finally, this paper verified the correctness of this inversion method by different shape defects. The results showed that this method has a good recognition effect on different shape defects, and strong generalization ability is its prominent advantage.

\section{References}

1. LS Peng, SL Huang, W Zhao. EMI, 52, $1-6(2015)$

2. YS Li, QZ Liu, J Liu. TCE, 32,176-185(2017)

3. WH Han, J Xu, XH Shen. FCCC,40, 88-91,(2015)

4. SL Huang, LS Peng, W Zhao. TCE, 31, 55-63(2016)

5. JL He, GP Wang, D Liu. PSPC, 45, 69-76(2017)

6. B Ma, YL Zhang. PSPC, 43, 78-82(2015)

7. L Wang, J Zhao, DC liu. TCE, 31, 204-210(2016) 